\title{
Linearized Error Analysis for an Accelerator and Application to the APS Injector Synchrotron
}

\author{
R. K. Koul and F. E. Mills \\ Argonne National Laboratory, 9700 South Cass Avenue, \\ Argonne, Il 60439.
}

\begin{abstract}
This paper presents a tolerance budget for accelerators dictated by the linear transverse dynamics of particle motion. The linearized equations satisfied by the particle motion when errors in the lattice are present are given along with the solution to these equations. The forms of these errors giving rise to the linearized equation are stated. These results are used to derive a tolerance budget for the Advanced Photon Source (APS) injector synchrotron.
\end{abstract}

\section{Introduction}

It is well known that the design of an accelerator differs from the operating conditions because of the idealized character of the forces used in the design stages. These deviations of idealized forces from actual forces acting on the particles in an accelerator will be called errors in all that follows. These errors in turn change the lattice functions of the idealized accelerator. If the deviations are large, there can be instabilities of the beam motion. Thus it is desirable to get some estimate of the effects of errors on the lattice parameters of an accelerator. These effects in turn dictate the tolerances one can have on the sources of these errors.

The error sources can be random or systematic. Some of the most common sources of errors are (a) random or systematic positional errors of the magnetic elements and (b) random or systematic multipole fields present in the magnetic elements.

This paper focuses on the linear dynamics only. The paper is divided into two sections and four appendices. The first section deals with the transverse equations of motion and the form of their solutions and attempts to qualitatively distinguish the main sources of error and their effects. The second section displays the equations satisfied when these errors are present and the general form of the solutions of these equations and the conditions required to obtain these solutions. Some comments are presented about the use of these expressions when the errors are systematic, and the expressions are simplified

This manuscript has been authored under contract number W31-109-ENG-38 with the U.S. Department of Energy. Accordingly, the U.S. Government retains a non-exclusive, royalty-free license to publish or reproduce the published form of this contribution, or allow others to do so, for U.S. Government purposes. 


\section{DISCLAIMER}

Portions of this document may be illegible in electronic image products. Images are produced from the best available original document. 
for use when errors are random. These two sections are followed by three appendices containing the derivation of the errors, the derivations of linearized equations of motion, and their solutions. Appendix D contains an application to the APS injector synchrotron and the resulting tolerances on the lattice parameters.

Some of this work is well known and can be found in references listed at the end. One of the purposes of this paper is to collect all this information in a single document for reference.

\section{Equations of Motion}

In the following we will assume that the accelerators are planar. The radius of curvature $\rho$ is piecewise constant and there is no energy change at the particle. We will neglect the coupled motion caused by sextupoles and skew quadrupoles. We will neglect all nonlinear contributions to the equations of motion. We will assume accelerator coordinates such that the line element is given by:

$$
d l^{2}=d x^{2}+d y^{2}+(1+x / \rho)^{2} d s^{2}
$$

Note that in the straight sections $\rho$ is infinite and $d s=d z$. It is straightforward to verify that in these coordinates the equations of motion take the following standard form (Edwards ${ }^{1}$ ):

$$
\begin{aligned}
x^{\prime \prime}-\left((\rho+x) / \rho^{2}\right) & =\left(-e B_{y} / p_{s}\right)(1+x / \rho)^{2} \\
y^{\prime \prime} & =\left(-e B_{x} / p_{s}\right)(1+x / \rho)^{2} .
\end{aligned}
$$

Because $B_{y}$ and $B_{x}$ are polynomials in $\mathrm{x}$ and $\mathrm{y}$ and the coefficients of these polynomials are functions of $s$, it is not known at present how to solve these equations analytically. At present we are not even interested in that question. These equations are simplified considerably in view of our previously stated assumptions. Considering only the linear part of the magnetic field ( see Courant ${ }^{2}$ or Michelotti ${ }^{3}$ or any of your favorite authors) we obtain the linear equations

$$
\begin{aligned}
x^{\prime \prime}+\left(1 / \rho^{2}-k\right) x & =(1 / \rho)\left(1-p_{0} / p_{s}\right), \\
y^{\prime \prime}+k y & =0 .
\end{aligned}
$$

If we further assume that the momentum $p_{s}$ is equal to $p_{0}$ we obtain the standard homogeneous equations satisfied for the transverse motion of the particles in an accelerator as:

$$
\begin{aligned}
x^{\prime \prime}+\left(1 / \rho^{2}-k\right) x & =0 \\
y^{\prime \prime}+k y & =0
\end{aligned}
$$

These equations are similar to the harmonic oscillator equations of a unit point mass whose spring constant (or what may be called frequency) depends on time. The spring constants in the above equations are the quadrupole strength and the inverse square of the curvature of the trajectory in the dipole. Its formal periodic solutions have been extensively studied. In the following, for the sake of the completion, 
we will write down the solutions. The interested reader can refer for details to Courant ${ }^{2}$ or Lichtenberg 4 or Steffen ${ }^{5}$, or any of several accelerator summer school proceedings.

Therefore, without any further details we will write down the transformations which bring these equations in the phase amplitude form and the equations satisfied by those variables. We define:

$$
\begin{gathered}
x(s)=W(s) U(s), W(s)=\beta^{1 / 2}, U(s)=A e^{\imath \Psi(s)}, \\
W^{\prime \prime}+K(s) W(s)-W^{-3}=0, \Psi=\int W^{-2} d s \\
\nu=(2 \pi)^{-1} \oint W^{-2} d s, \phi=1 / \nu \int W^{-2} d s
\end{gathered}
$$

where $W(s)$ is periodic in s with some period $\delta s$ and $\Psi(s+\delta s)-\Psi(s)=\delta \Psi . \delta \psi$ is the phase advance over the distance $\delta s$. The equation of motion takes the following simple form:

$$
\frac{d^{2} U}{d \phi^{2}}+\nu^{2} U=0
$$

\section{Linearized Equation in the Presence of Errors}

The Eq. (1.11) is the equation for a simple harmonic oscillator for the variable $U$ as a function of the parameter $\phi$, with $\nu$ the natural frequency. However, the deceptively simple-looking equation has all the complications hidden in Eq. (1.9). Notice that frequency $\nu$ is given by equation Eq. (1.10) and that the equation can be evaluated only if equation Eq. (1.9) for $\mathrm{W}(\mathrm{s})$ can be solved. One of the driving terms in that equation is the quadrupole strength parameter $\mathrm{K}(\mathrm{s})$ from Eq. (1.6) or Eq. (1.7). Therefore, errors in the quadrupole strength of the equations of motion will change the frequency $\nu$ and the amplitude function $\beta$. The source of error in the quadrupole strength can be the result of errors in the quadrupole field, or other multipole content present in magnets, or tilts and/or displacement of magnetic elements with higher-order multipole fields. Quadrupole strength is not the only parameter which can differ between the design and the actual machine. In the equations of motion above, the dipole field is designed to give a constant radius of curvature, $1 / \rho$. However, in practice the dipole field of the bending magnet can vary from the desired goal, giving rise to errors in the bending radius. Additional sources of bending field errors can come from tilt in the bending magnets or positional displacement of the quadrupoles, sextupoles, etc. Other very important sources of errors are quadrupole displacements and tilts in the bending magnets. All these dipole field errors appear as constant driving terms on the right-hand sides of Eq. (1.6) and Eq. (1.7). These source terms give rise to orbit distortions. Lastly, recall that in obtaining the linear homogeneous equation we assumed that the actual momentum of the particle is the same as the nominal momentum assumed in defining $1 / \rho=(e B) /\left(c P_{0}\right)$. If the momentum $p_{s}$ is different from the nominal momentum, then there are source terms which are proportional to the momentum deviation from the nominal momentum, causing orbit dispersions. In the following section we treat the linearized equations to be satisfied in the presence of different sources of errors. 


\section{ORBIT DISTORTION:}

First of all consider the bend field errors. The linearized equations of motion are written as:

$$
\begin{aligned}
& X^{\prime \prime}+K_{x}(s) X=f_{x}(s) \\
& Y^{\prime \prime}+K_{y}(s) Y=f_{y}(s)
\end{aligned}
$$

The formal structure of both the $\mathrm{X}$ and the $\mathrm{Y}$ equations of motion given above is the same. So it will be sufficient if we deal with only one of them. The form of the source function $f_{x}(s)$ and $f_{y}(s)$ is given in Appendix C.

If we again introduce the variables $U$ and $\phi$ as defined in Eq. (1.8) through Eq. (1.10) and use these in Eq. (2.1) or Eq. (2.2) we obtain:

$$
\frac{d^{2} U}{d \phi^{2}}+\nu^{2} U=-\nu^{2} \beta^{3 / 2} f(\phi), f(\phi)=f(\phi(s))
$$

The solution to the homogeneous equation obtained from Eq. (2.3) is characterized by the invariant function $I=U^{2}+1 / \nu^{2} \frac{d U^{2}}{d \phi}$ which is a constant of motion. In fact this $I$ is just the square of the A term given in Eq. (1.8). The solution of the inhomogeneous equation can be obtained by the Green function method from the linear combinations of the known solutions of the homogeneous equation. We demand that the Green function satisfy the periodic boundary conditions. Also we demand that the Green function be continuous at the source point and its derivative be discontinuous at the source point. (See details in Appendix B.) With the above procedure, the Green function and the solution are respectively given by

$$
\begin{aligned}
& G\left(\phi, \phi^{\prime}\right)=\frac{\cos \nu\left(\phi-\phi_{i}+\pi\right)}{(2 \sin \pi \nu)} \\
& U_{p}(\phi)=-\frac{\nu}{(2 \sin (\pi \nu))} \int_{\phi}^{\phi+2 \pi} \beta^{3 / 2} f\left(\phi_{i}\right) \cos \nu\left(\phi-\phi_{i}+\pi\right) d \phi_{i},
\end{aligned}
$$

where $\phi_{i}$ is the position of the source term. From the solution of Eq. (2.5) we can construct the invariant I, the square of the amplitude function in terms of which we can express the transverse orbit coordinates and slopes, for the orbit distortions in $\mathrm{X}$ or $\mathrm{Y}$ at any point along the ring are given by $\sqrt{I}$. Using the above expression for I, we get

$$
I=\left(\frac{\nu}{(2 \sin \pi \nu)}\right)^{2} \int_{\phi}^{\phi+2 \pi} d \phi_{2} \int_{\phi}^{\phi+2 \pi} d \phi_{1} \beta^{3 / 2}\left(\phi_{1}\right) \beta^{3 / 2}\left(\phi_{2}\right) f\left(\phi_{1}\right) f\left(\phi_{2}\right) \cos \nu\left(\phi_{2}-\phi_{1}\right) .
$$

The invariant is independent of the phase angle $\phi$ and depends only on the phase difference between different sources, as it should be. Since the error sources are distributed among the discrete magnetic elements, the double integral over the phase reduces to the double sum over the magnetic elements over which $f(\phi)$ is non-zero with integration to be carried out over the length of the element itself. Notice that the beta functions and the tune in the integral are the parameters for the perfect machine. Therefore, changing the above equation to the double sum form we get,

$$
I=\frac{\nu}{(2 \sin \pi \nu)^{2}} \sum_{i=(\text { element }}^{i=(\text { element }} \int_{0}^{L_{i}} d \phi_{i} \sum_{j=(\text { element }}^{j=(\text { element }} \int_{0}^{L_{j}} d \phi_{j}\left[f_{i} f_{j}\left(\beta_{i} \beta_{j}\right)^{3 / 2} \cos \nu\left(\phi_{i}-\phi_{j}\right)\right]
$$


If we furthermore use the fact that $d \phi=(\nu \beta)^{-1} d s$ and the fact that over an element the $d s=d l$ we get

$$
I=\frac{1}{(2 \sin \pi \nu)^{2}} \sum_{i=(\text { element }}^{i=\left(\text { element }_{j}\right.} \int_{0}^{L_{i}} d l_{i} \sum_{j=(\text { element }}^{j=\left(\text { element }_{\lambda}\right.} \int_{0}^{L_{j}} d l_{j}\left[f_{i} f_{j}\left(\beta_{i} \beta_{j}\right)^{1 / 2} \cos \nu\left(\phi_{i}-\phi_{j}\right)\right] .
$$

We thus have in Eq. (2.8) the expression for the amplitude factor as a function of the errors at position $\phi_{i}$ and the corresponding beta functions at the position of the error and the cosine of the phase difference between different error positions. The amplitude factor is symmetric in the interchange of the indices $i$ and $\mathrm{j}$ which refer to the location of the error sources.

We can simplify Eq. (2.8) by assuming that $\phi$ does not vary significantly over the element under consideration. We get:

$$
I=\left(\frac{1}{(2 \sin \pi \nu)}\right)^{2} \sum_{i=(\text { element } j j=(\text { element }}^{i=(\text { element })_{j} j=(\text { element }} L_{i} L_{j}\left[f_{i} f_{j}\left(\beta_{i}\right)^{1 / 2}\left(\beta_{j}\right)^{1 / 2} \cos \nu\left(\phi_{i}-\phi_{j}\right)\right] .
$$

Eq. (2.9) can be used to find the amplification factors if we know the sources of the error. In the case of systematic errors we just carry out the double sum using the error distribution. However, in the case of random distribution the double sum reduces to a single sum, i.e only contributions to the rms amplification factor are for $i=j$. In that case we have

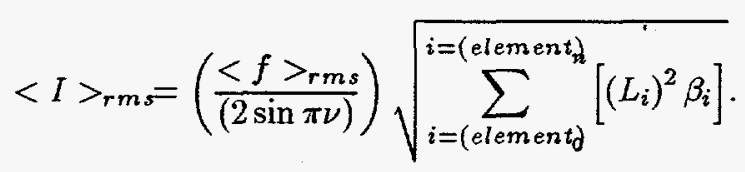

For applications, a comprehensive list of the error sources $f(\phi)$ is provided in Appendix C.

\section{GRADIENT ERRORS:}

As stated in the beginning of Section 2, the coefficient $K$ in the equations of motion is different from $\hat{K}$ for the real machine. In this case one would like to know the deviation of the orbit parameters between the real machine and the designed one. Recall that $K$ in the equations of motion defines $W(s)=\beta^{\frac{1}{2}}$ through Eq. (1.9) and $\nu$ through Eq. (1.10). Thus, any change in K would change both the $\beta$ function and the tune $\nu$. In the following we assume that the solution of the equation for $W(s)$ is for the designed $K(s)$, and we obtain the equations of motion linearized off this solution. To that end we assume that:

$$
\hat{K}=K+\delta K, \quad \hat{W}(s)=W(s)+\delta W, \quad \hat{W}(s)=W(s)\left\{1+\frac{\delta W}{W}\right\} .
$$

Since $\hat{W}(s)$ satisfies the equation analogous to Eq. (1.8) we use the right-hand side of Eq. (2.11) in it. After writing the equation in terms of the variables $\phi$ and $\delta W / W$, we obtain the following (see Appendix A for details of the derivation):

$$
\begin{gathered}
\frac{d^{2}}{d \phi^{2}}\left(\frac{\delta W}{W}\right)+4 \nu^{2}\left(\frac{\delta W}{W}\right)=F(\phi) \\
F(\phi)=-\nu^{2} W^{4} \delta K
\end{gathered}
$$

Notice that Eq. (2.12) for $\frac{\delta W}{W}$ satisfies the equation for the driven harmonic oscillator with frequency twice the frequency of the corresponding orbit distortion equation, Eq. (2.3), the driving term being 
proportional to the square of the unperturbed $\beta$ function. Associated with this equation is the invariant, say $I_{\delta \beta}$ (see Appendix B for details), which is the square of the amplitude of the $\left(\frac{\delta W}{W}\right)$ oscillations. Again, we solve Eq. (2.12) subject to the conditions of the periodicity, with periodicity given by the revolution time period of the particle. In addition, we demand the continuity of the Green function at the source point and the discontinuity in the derivative of the Green function equal to the strength of the source term. We obtain

$$
\begin{aligned}
G_{\delta W}\left(\phi, \phi_{1}\right) & =\frac{\cos 2 \nu\left(\phi-\phi_{1}+\pi\right)}{4 \nu \sin (2 \pi \nu)}, \\
\quad \frac{\delta W}{W} & =\frac{1}{4 \nu \sin (2 \pi \nu)} \int_{\phi}^{\phi+2 \pi} F\left(\phi_{1}\right) \cos 2 \nu\left(\phi-\phi_{1}+\pi\right) d \phi_{1} .
\end{aligned}
$$

The corresponding invariant function $I_{\delta \beta}$, can be written as follows:

$$
I_{\delta \beta}=\left(\frac{\delta W}{W}\right)^{2}+\left(\frac{1}{2 \nu}\right)^{2}\left(\left(\frac{\delta W}{W}\right)^{\prime}\right)^{2}
$$

Using Eq. (2.15) in Eq. (2.16) we obtain,

$$
I_{\delta \beta}=\left(\frac{1}{4 \nu \sin (2 \pi \nu)}\right)^{2} \int_{\phi}^{\phi+2 \pi} d \phi_{2} \int_{\phi}^{\phi+2 \pi} F\left(\phi_{1}\right) F\left(\phi_{2}\right) \cos 2 \nu\left(\phi_{1}-\phi_{2}\right) d \phi_{1} .
$$

In a given accelerator, the sources of the error $F(\phi)$ are distributed discreetly around the ring in the attributes of the magnetic elements. Hence, in any particular application the function $F(\phi)$ vanishes for most values of $\phi$ except at a finite number of values of $\phi$. We can then replace the double integration over $\phi$ by a double sum with integration carried over the region of the magnetic element which is the source of the error. Under these conditions we get

$$
I_{\delta \beta}=\left(\frac{1}{4 \nu \sin (2 \pi \nu)}\right)^{2} \sum_{i=0}^{i=n} \int_{0}^{L_{i}} d \phi_{i} \sum_{j=0}^{j=n} \int_{0}^{L_{j}} d \phi_{j}\left[F\left(\phi_{i}\right) F\left(\phi_{j}\right) \cos 2 \nu\left(\phi_{i}-\phi_{j}\right)\right] .
$$

We can further simplify Eq. (2.18) by changing the integration variable from $\phi$ to $s$ using $\nu d \phi=\beta^{-1} d s \simeq$ $\beta^{-1} d l$ over the magnetic element. We also assume that in $F\left(\phi_{i}\right)=\nu^{2} W_{i}^{4} \delta K\left(\phi_{i}\right), \delta K\left(\phi_{i}\right)$ is constant over the length of the i'th element. Consequently we can replace $\delta K\left(\phi_{i}\right)$ by $\delta K_{i}$ :

$$
\begin{aligned}
I_{\delta \beta} & =\left(\frac{1}{4 \sin (2 \pi \nu)}\right)^{2} \sum_{i=0}^{i=n} \int_{0}^{L_{i}} d l_{i} \sum_{j=0}^{j=n} \int_{0}^{L_{j}} d l_{j} \beta_{i} \beta_{j}\left[\delta K_{i} \delta K_{j} \cos 2 \nu\left(\phi_{i}-\phi_{j}\right)\right], \\
\delta K_{i} & =\frac{\delta B_{i}{ }^{\prime}}{B^{\prime}} K_{i}=\frac{\delta B_{i}^{\prime}}{B \rho}
\end{aligned}
$$

We need to make one more assumption in order to render the above expression simple for applications to accelerators: that the phase $\phi_{i}$ does not vary over the length of the magnetic element. Under this condition the integration over $d l_{i}$ contributes the length of the magnetic element, and we have

$$
I_{\delta \beta}=\left(\frac{1}{4 \sin (2 \pi \nu)}\right)^{2} \sum_{i=\left(\text { element } t_{j} j=\left(\text { element } t_{j}\right.\right.}^{i=\left(\text { element } t_{\eta} j=\left(\text { element } t_{\lambda}\right.\right.} L_{i} L_{j} \beta_{i} \beta_{j}\left[\delta K_{i} \delta K_{j} \cos 2 \nu\left(\phi_{i}-\phi_{j}\right)\right]
$$


The Eq. (2.21) is the invariant function associated with the linearized equation of the motion for $\delta \beta / \beta$, and is, in fact, the square of the amplitude of the motion. In terms of $I_{\frac{\delta \beta}{\beta}}$ we have

$$
\frac{\delta \beta}{\beta} \approx \sqrt{I_{\delta \beta}} \operatorname{Sin} 2(\nu \phi)
$$

If the sources of our errors are systematic we can carry out the double sum and find the change in the $\beta$ function. In case of random errors we can either find the rms value of the change in $\beta$ or carry out the double sum and associate the invariant amplitude function with $\delta \beta / \beta$. If we are only interested in the root mean square value of the double sum the expression becomes particularly simple:

$$
\begin{aligned}
& <I_{\delta \beta}>_{r m s}=\left(\frac{<\delta K>_{r m s}}{2 \sin (2 \pi \nu)}\right)^{2} \sum_{i=(\text { element }}^{i=(\text { element }}{ }_{i}{ }_{i}{ }^{2} \beta_{i}{ }^{2}, \\
& <\frac{\delta \beta}{\beta}>_{r m s}=\frac{\left\langle\delta K>_{r m s}\right.}{2 \sin (2 \pi \nu)} \sqrt{\sum_{i=(\text { element }}^{i=\left(\text { element } t_{i}\right.} L_{i}^{2} \beta_{i}^{2}} .
\end{aligned}
$$

In obtaining Eq. (2.24) for $\frac{\delta \beta}{\beta}$ we have used the relation $W^{2}=\beta$. Notice again that Eq. (2.12) satisfied by $\delta \beta / \beta$ is one of simple harmonic motion. And $\delta \beta / \beta$ is maximum when $(\delta \beta / \beta)^{\prime}$ vanishes. Hence $\max (\delta \beta(s) / \beta(s))=I_{\delta \beta}$. Therefore, knowing $\beta(s)$ and $I_{\delta \beta}$ tells us the maximum value $\delta \beta$ can have at any place in the accelerator ring.

Since the tune is given by $\nu=\frac{1}{2 \pi} \int_{0}^{2 \pi} \beta^{-1} d s$, we can obtain the change in tune $\delta \nu$, from the change in $\beta$. The expression for the change in tune $\Delta \nu / \nu$ can be obtained by using Eq. (2.22) in the expression for $\nu$ :

$$
\frac{\Delta \nu}{\nu}=\frac{1}{4 \pi \nu} \sqrt{\sum_{i=\left(\text { element } t_{j}\right.}^{i=\left(\text { element } t_{\eta} j=\left(\text { element } t_{j}\right.\right.} \sum_{i} \delta k_{i} \delta k_{j} L_{j} \beta_{i} \beta_{j} \operatorname{Cos} 2 \nu\left(\phi_{i}-\phi_{j}\right)}
$$

\section{MOMENTUM SPREAD EFFECTS:}

Recall that in obtaining the linear equations of motion we assumed that the longitudinal momentum used for defining the magnetic rigidity is the same as $p_{s}$. In general, the particle bunch has a longitudinal momentum spread. Particles of momentum $p_{s} \neq p_{0}$ are said to have a momentum error. We will next investigate the trajectory of these off-momentum particles. Again, starting from the linear equation of motion Eq. (1.6), using $p_{s}=P_{0}+\Delta p$ and expanding $\left(p_{0}+\Delta p\right)^{-1}$ in the power series and keeping only the linear terms we obtain

$$
X^{\prime \prime}+k(s) X=F_{1}(s)+\frac{\Delta p}{p} F_{2}(s), F_{2}(s)=1 / \rho+f(s) .
$$

We will demand that the solution of Eq. (2.26) be of the form $X_{\beta}+D \frac{\Delta p}{p}$ such that

$$
\begin{aligned}
& X_{\beta}{ }^{\prime \prime}+k(s) X_{\beta}=F_{1}(s), \\
& D^{\prime \prime}+k(s) D=1 / \rho+f(s) .
\end{aligned}
$$

The solution of the first of the above equations has already been considered previously. Here we are interested in Eq. (2.27). 
If $f(s)$ in Eq. (2.27) vanishes, we obtain an equation with the source term given by $1 / \rho$, which is the well-known source term for driving the dispersion effects. We will next assume that the solution of the equation with $f(s)=0$ is already known and is given by $D(s)$. Hence we can assume that for $f(s)<1 / \rho$ the solution is given by $D(s)+\Delta D(s)$. Inserting this into Eq. (2.27) we obtain the equation satisfied by $\Delta D(s)$ :

$$
\Delta D^{\prime \prime}+k(s) \Delta D=-k_{n} D+f(s) .
$$

The coefficient $k_{n}$ of $\mathrm{D}$, the first term on the right-hand side of the above equation, is proportional to $\frac{1}{B \rho}$ times the alignment and field errors in the magnetic elements. Its form is given in Appendix C.

We will proceed in a similar manner, assuming that $\Delta D=\sqrt{\beta} U(\phi)$, where $d \phi=\frac{1}{\nu \beta}$. Inserting these in Eq. (2.28) and using $2 \beta \beta^{\prime \prime}-\beta^{2}+4 \beta^{2} k=4$, we get (see Appendix A),

$$
\frac{d^{2} U}{d \phi^{2}}+\nu^{2} U=-\nu^{2} \beta^{3 / 2}\left[k_{n} D+f(s)\right] \text {. }
$$

Once again notice that Eq. (2.29) is the linear equation of the driven harmonic oscillator with frequency $\nu$. Associated with the above equation is the invariant $I_{\delta D}=U^{2}+\frac{1}{\nu^{2}}\left(\frac{d U}{d \phi}\right)^{2}$, where $U(\phi)$ is the solution of the inhomogeneous equation, Eq. (2.29). The solution $U(\phi)$ satisfying the periodic orbit boundary conditions can be written as follows:

$$
U(\phi)=\frac{1}{2 \nu \sin (\pi \nu)} \int_{\phi}^{\phi+2 \pi} \nu^{2} \beta\left(\phi_{1}\right)^{3 / 2} f\left(\phi_{1}\right) \cos \nu\left(\phi-\phi_{1}+\pi\right) d \phi_{1} .
$$

In terms of the solution Eq. (2.30) we have

$$
I_{\delta D}=\left(\frac{1}{2 \nu \sin (\pi \nu)}\right)^{2} \int d \phi_{2} \int d \phi_{1} f\left(\phi_{1}\right) \nu^{4} \beta\left(\phi_{1}\right)^{3 / 2} \beta\left(\phi_{2}\right)^{3 / 2} f\left(\phi_{2}\right) \cos \nu\left(\phi_{1}-\phi_{2}\right)
$$

Again the above expression is simplified under the assumption that sources of error are distributed discreetly around the ring in the attributes of the magnetic elements, and the integral contributes only at those elements. Under these assumptions we get

$$
I_{\delta D}=\left(\frac{\nu}{2 \sin (\pi \nu)}\right)^{2} \sum_{i=0}^{i=n} \int_{0}^{L_{i}} d \phi_{i} \sum_{j=0}^{j=n} \int_{0}^{L_{j}} d \phi_{j} f\left(\phi_{i}\right)\left(\beta\left(\phi_{i}\right) \beta\left(\phi_{j}\right)\right)^{3 / 2} f\left(\phi_{j}\right) \cos \nu\left(\phi_{i}-\phi_{j}\right) .
$$

Furthermore, using $\nu d \phi=\beta^{-1} d s$ and $d s=d L_{i}$ over the i'th element and assuming that $\beta$ changes very little within the magnetic element, we can write

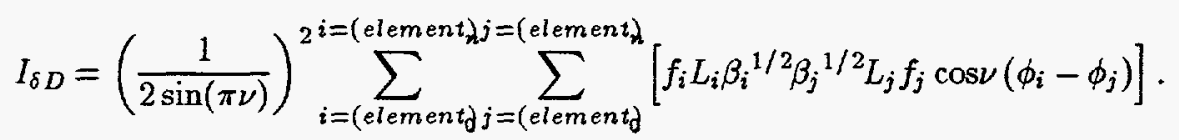

In the above form we have carried the integration over the magnetic element and assumed the value of $\phi_{i}$ is the value at the center of the magnetic element. If the error sources are assumed to be random in character we can estimate the root mean square value of the "invariant" amplification factor $I_{\delta D}$ from Eq. (2.33) (see Appendix for the most commonly used forms of I):

$$
<I_{\delta D}>_{r m s}=\frac{<f>_{r m s}}{2 \sin (\pi \nu)} \sum_{i=(\text { element }}^{i=\left(e \text { element } t_{\mathfrak{d}}\right.} L_{i}^{2} \beta_{i}
$$




\section{Appendix A.}

\section{Sources of Error}

\section{ERROR SOURCES:}

In this appendix we will give the explicit form of the sources of errors which contribute as sources of the linear equation of motion. We will assume magnetic multipole fields up to octupoles, introduce the field errors and the placement errors of the magnetic elements along with the momentum errors of the particle. After that we will obtain the equations where these errors contribute as sources.

In the following we will assume that (a) the accelerator is planar, (b) the radius of curvature $\rho$ is piecewise constant, (c) there is no energy transfer to the particle or energy loss by the particle, (d) the coupled motion caused by the sextupole type of coupling or skew quadrupole type of coupling is neglected, (e) all the nonlinear contributions to the equations of motion are ignored, and (f) the accelerator coordinates are such that the line element is given by

$$
d l^{2}=d x^{2}+d y^{2}+(1+x / \rho)^{2} d s^{2}
$$

Note that in the straight sections $\rho$ is infinite and $d s=d z$. Also in what follows B will stand for $B_{y}$ or $B_{x}$.

As stated earlier we have assumed, (g) the magnetic field has terms up to order three, in multipole expansion:

$$
B=B_{0}+B^{\prime} x+\frac{x^{2}}{2 !} B^{\prime \prime}+\frac{x^{3}}{3 !} B^{\prime \prime \prime},
$$

and $(\mathrm{h})$ in the perturbative expansion of the field errors we will keep at most second-order terms in the errors.

Under field errors, the dipole part of the field is $B_{0}+\Delta B_{0}$, the quadrupole part is $B^{\prime}+\Delta B^{\prime}$, the sextupole part is $B^{\prime \prime}+\Delta B^{\prime \prime}$, and the octupole part is $B^{\prime \prime \prime}+\Delta B^{\prime \prime \prime}$. Under displacement error $\Delta x$, the position of the centerline of the magnet changes to $x+\Delta x$. Using these expressions for the magnetic field we can write

$$
B=\left(B_{0}+\Delta B_{0}\right)+(x+\Delta x)\left(B^{\prime}+\Delta B^{\prime}\right)+\frac{(x+\Delta x)^{2}}{2 !}\left(B^{\prime \prime}+\Delta B^{\prime \prime}\right)+\frac{(x+\Delta x)^{3}}{3 !}\left(B^{\prime \prime \prime}+\Delta B^{\prime \prime \prime}\right) .
$$

Taking $B_{0}$ as a common factor we obtain

$$
\begin{aligned}
B= & B_{0}\left[1+\frac{\Delta B_{0}}{B_{0}}+\frac{B^{\prime}}{B_{0}}\left(1+\frac{\Delta B^{\prime}}{B^{\prime}}\right)(x+\Delta x)+\frac{(x+\Delta x)^{2}}{2 !} \frac{B^{\prime \prime}}{B_{0}}\left(1+\frac{\Delta B^{\prime \prime}}{B^{\prime \prime}}\right)\right]+ \\
& B_{0}\left[\frac{(x+\Delta x)^{3}}{3 !} \frac{B^{\prime \prime \prime}}{B_{0}}\left(1+\frac{\Delta B^{\prime \prime \prime}}{B^{\prime \prime \prime}}\right)\right] .
\end{aligned}
$$

The equation of motion as in Eq. (1.2) (see section 1 on Equations of Motion) has term $\frac{e B}{p_{s}}\left(1+\frac{x}{\rho}\right)^{2}$. In the following we will assume that the momentum $p_{s}=p_{0}+\Delta p$, where $p_{0}$ is the nominal momentum of 
the particle on the designed trajectory. We will further assume that:

$$
\frac{-B^{\prime}}{B_{0} \rho}=k, \quad \frac{B^{\prime \prime}}{B_{0} \rho}=\mathbf{s}, \quad \frac{B^{\prime \prime \prime}}{B_{0} \rho}=O .
$$

Using the definitions in Eq. (A.5) and the form of $p_{s}$ assumed earlier, we can write the right side of Eq. (1.2) as follows:

$$
\begin{gathered}
\frac{e B}{p_{s}} \simeq\left(1-\frac{\Delta p}{p}\right)\left[\frac{1}{\rho}+\left\{\frac{\Delta B_{0}}{B_{0} \rho}-k\left(1+\frac{\Delta B^{\prime}}{B^{\prime}}\right) \Delta x+\frac{\mathrm{s}}{2}\left(1+\frac{\Delta B^{\prime \prime}}{B^{\prime \prime}}\right) \Delta x^{2}\right\}\right]+ \\
\left(1-\frac{\Delta p}{p}\right)\left[(-k)\left(1+\frac{\Delta B^{\prime}}{B^{\prime}}\right)+\mathrm{s}\left(1+\frac{\Delta B^{\prime \prime}}{B^{\prime \prime}}\right) \Delta x+\frac{O}{2}\left(1+\frac{\Delta B^{\prime \prime \prime}}{B^{\prime \prime \prime}}\right) \Delta x^{2}\right] x+ \\
\left(1-\frac{\Delta p}{p}\right)\left[\left[\frac{\mathrm{s}}{2}\left(1+\frac{\Delta B^{\prime \prime}}{B^{\prime \prime}}\right)+\frac{O}{2}\left(1+\frac{\Delta B^{\prime \prime \prime}}{B^{\prime \prime \prime}}\right) \Delta x\right] x^{2} \frac{O}{3 !}\left(1+\frac{\Delta B^{\prime \prime \prime}}{B^{\prime \prime \prime}}\right) \Delta x^{3}\right] .
\end{gathered}
$$

In the following we will ignore the coefficients of the nonlinear terms (i.e $x^{2}$ terms) and also the terms of the form $\Delta x^{3}$ in the right-hand side of Eq. (1.2), i.e third-order terms in the perturbation expansion.

$$
\begin{gathered}
\frac{e B}{p_{s}}\left(1+\frac{x}{\rho}\right)^{2} \simeq\left(1+\frac{2 x}{\rho}\right)\left[\left(1-\frac{\Delta p}{p}\right)\left[\rho^{-} 1+\left\{\frac{\Delta B_{0}}{B_{0} \rho}-k\left(1+\frac{\Delta B^{\prime}}{B^{\prime}}\right) \Delta x+\frac{s}{2} \Delta x^{2}\right\}\right]\right]+ \\
\left(1-\frac{\Delta p}{p}\right)\left[(-k)\left(1+\frac{\Delta B^{\prime}}{B^{\prime}}\right)+\mathrm{s}\left(1+\frac{\Delta B^{\prime \prime}}{B^{\prime \prime}}\right) \Delta x+\frac{O}{2} \Delta x^{2}\right] x .
\end{gathered}
$$

Next, separating the terms which are coefficients of the $\mathrm{x}$ and the constant terms, we get

$$
\begin{aligned}
\frac{e B}{p_{s}}\left(1+\frac{x}{\rho}\right)^{2} & \simeq\left(1-\frac{\Delta p}{p}\right)\left[\frac{1}{\rho}+\left\{\frac{\Delta B_{0}}{B_{0} \rho}-k\left(1+\frac{\Delta B^{\prime}}{B^{\prime}}\right) \Delta x+\frac{\mathbf{s}}{2} \Delta x^{2}\right\}\right]+ \\
& \left(1-\frac{\Delta p}{p}\right)\left[\left\{-k\left(1+\frac{\Delta B^{\prime}}{B^{\prime}}\right)+\mathrm{s}\left(1+\frac{\Delta B^{\prime \prime}}{B^{\prime \prime}}\right) \Delta x+\frac{O}{2} \Delta x^{2}\right\}\right]+ \\
& \left(1-\frac{\Delta p}{p}\right)\left[\frac{2}{\rho}\left\{\frac{1}{\rho}+\frac{\Delta B_{0}}{B_{0} \rho}-k\left(1+\frac{\Delta B^{\prime}}{B^{\prime}}\right) \Delta x+\frac{\mathbf{s}}{2} \Delta x^{2}\right\}\right] x
\end{aligned}
$$

We define:

$$
\begin{aligned}
& f_{\text {ditort }}=-\left\{\frac{\Delta B_{0}}{B_{0} \rho}+(-k)\left(1+\frac{\Delta B^{\prime}}{B^{\prime}}\right) \Delta x+\frac{\mathrm{s}}{2} \Delta x^{2}\right\} \\
& f_{\text {dispers }}=\left\{\frac{\Delta B_{0}}{B_{0} \rho}+(-k)\left(1+\frac{\Delta B^{\prime}}{B^{\prime}}\right) \Delta x+\frac{\mathrm{s}}{2} \Delta x^{2}\right\} \\
& \delta k_{\beta}=\left[\left\{-k \frac{\Delta B^{\prime}}{B^{\prime}}+\mathrm{s}\left(1+\frac{\Delta B^{\prime \prime}}{B^{\prime \prime}}\right) \Delta x+\frac{O}{2} \Delta x^{2}\right\}+\frac{2}{\rho}\left\{\frac{\Delta B_{0}}{B_{0} \rho}-k\left(1+\frac{\Delta B^{\prime}}{B^{\prime}}\right) \Delta x+\frac{\mathbf{s}}{2} \Delta x^{2}\right\}\right] .(A)
\end{aligned}
$$

Using the above definitions in Eq. (A.7) for the right-hand side of Eq. (1.2) we get

$$
\begin{aligned}
\frac{e B}{p_{s}}\left(1+\frac{x}{\rho}\right)^{2} \simeq & \rho^{-1}-f_{\text {ditort }}-\frac{\Delta p}{p}\left[\rho^{-1}+f_{\text {disper }}\right]+ \\
& {\left[\left(2 \rho^{-2}-k\right)+\delta k_{\beta}-\frac{\Delta p}{p}\left\{\left(2 \rho^{-2}-k\right)+\delta k_{\beta}\right\}\right] x . }
\end{aligned}
$$

Both Eq. (A.12) and Eq. (A.8) describe the right-hand side of the equation of motion of the particles in the presence of errors. 


\section{Appendix B.}

\section{Equations of Motion}

\section{Equations for orbit distortion and dispersion:}

In order to derive the linear equations of motion of particles with forces specified by the error sources in Appendix A, we start with Eq. (1.2):

$$
\begin{aligned}
x^{\prime \prime}-\left((\rho+x) / \rho^{2}\right) & =\left(-e B_{y} / p_{s}\right)(1+x / \rho)^{2}, \\
y^{\prime \prime} & =\left(-e B_{x} / p_{s}\right)(1+x / \rho)^{2} .
\end{aligned}
$$

Using Eq. (A.12) for the right-hand side of the above equations, while keeping in mind that for the y-motion $\rho^{-1}=0$,

$$
\begin{aligned}
\left((\rho+x) / \rho^{2}\right)-\left(e B_{y} / p_{s}\right)(1+x / \rho)^{2} \simeq & +f_{\text {distort }}-\frac{\Delta p}{p}\left[\rho^{-1}+f_{\text {disperd }}\right]+ \\
& {\left[\left(k-\rho^{-2}\right)-\delta k_{\beta}+\frac{\Delta p}{p}\left\{\left(2 \rho^{-2}-k\right)+\delta k_{\beta}\right\}\right] x . }
\end{aligned}
$$

Using Eq. (B.3) in Eq. (B.1) and Eq. (B.2), and defining $K=\left(\rho^{-2}-k\right)$ we get

$$
\begin{aligned}
x^{\prime \prime}+\left[K+\delta k_{\beta}-\frac{\Delta p}{p}\left\{\left(2 \rho^{-2}-k\right)+\delta k_{\beta}\right\}\right] x & =f_{\text {distort }}+\frac{\Delta p}{p}\left[\rho^{-1}+f_{\text {disperd }}\right] \\
y^{\prime \prime}+\left[k+\delta k_{\beta}-\frac{\Delta p}{p}\left\{\left(2 \rho^{-2}-k\right)+\delta k_{\beta}\right\}\right] y & =f_{\text {distort }}+\frac{\Delta p}{p} f_{\text {dispers }}
\end{aligned}
$$

Because of the similarity of the above two equations we treat only Eq. (B.4). Let us assume that the solution of this equation is of the form $\bar{x}=\tilde{x}+\tilde{x}_{d i s p}$ such that,

$$
\begin{aligned}
\tilde{x}^{\prime \prime}+\left[K+\delta k_{\beta}-\frac{\Delta p}{p}\left\{\left(2 \rho^{-2}-k\right)+\delta k_{\beta}\right\}\right] \tilde{x} & =f_{\text {distort }} \\
\tilde{x}_{d i s p}^{\prime \prime}+\left[K+\delta k_{\beta}-\frac{\Delta p}{p}\left\{\left(2 \rho^{-2}-k\right)+\delta k_{\beta}\right\}\right] \tilde{x}_{d i s p} & =\frac{\Delta p}{p}\left[\rho^{-1}+f_{d i s p e r}\right] .
\end{aligned}
$$

Furthermore let us assume that $x_{d i s p}=\frac{\Delta p}{p} D$, implying thereby that $\bar{x}=\tilde{x}+\frac{\Delta p}{p} \tilde{D}$. Using our new substitution in Eq. (B.7) we obtain,

$$
\tilde{D}^{\prime \prime}+\left[K+\delta k_{\beta}-\frac{\Delta p}{p}\left\{\left(2 \rho^{-2}-k\right)+\delta k_{\beta}\right\}\right] \tilde{D}=\left[\rho^{-1}+f_{\text {disper }}\right\} \text {. }
$$

Notice that the coefficients of the linear parts of Eq. (B.6) and Eq. (B.8) are both harmonic oscillator type equations with the same $s$ dependent quadrupole strength. Also notice that when there are no magnetic field imperfections and no misalignment, the dispersion equation has the driving term given by $\rho^{-1}$. Let us consider the leading order terms in both these equations. If $\tilde{D}=D+\Delta D$ and $\tilde{x}=x+\Delta x$, where $D$ and $x$ satisfy Eq. (B.8) to the leading order in the expansion, in the absence of field and placement errors we have

$$
\begin{aligned}
x^{\prime \prime}+K x & =0, \\
D^{\prime \prime}+K D & =\rho^{-1} .
\end{aligned}
$$


The two perturbative parts $\Delta x$ and $\Delta D$ satisfy:

$$
\begin{aligned}
\Delta x^{\prime \prime}+\left[K+\delta k_{\beta}-\frac{\Delta p}{p}\left\{\frac{2}{\rho^{2}}-k+\delta k_{\beta}\right\}\right] \Delta x & =f_{\text {distort }}+\left[\delta k_{\beta}-\frac{\Delta p}{p}\left\{\frac{2}{\rho^{2}}-k+\delta k_{\beta}\right\}\right] x \\
\Delta D^{\prime \prime}+\left[K+\delta k_{\beta}-\frac{\Delta p}{p}\left\{\frac{2}{\rho^{2}}-k+\delta k_{\beta}\right\}\right] \Delta D & =f_{\text {dispers }}+\left[\delta k_{\beta}-\frac{\Delta p}{p}\left\{\frac{2}{\rho^{2}}-k+\delta k_{\beta}\right\}\right] D
\end{aligned}
$$

If we ignore terms higher than order one in Eq. (B.11) and higher than order two in Eq. (B.12) we obtain the following equations for $\Delta x$ and $\Delta D$, respectively:

$$
\begin{aligned}
\Delta x^{\prime \prime}+K \Delta x & =f_{\text {distort }}+\left[\delta k_{\beta}-\frac{\Delta p}{p}\left\{\left(2 \rho^{-2}-k\right)\right\}\right] x, \\
\Delta D^{\prime \prime}+K \Delta D & =f_{\text {dispers }}+\left[\delta k_{\beta}-\frac{\Delta p}{p}\left\{\left(2 \rho^{-2}-k\right)\right\}\right] D .
\end{aligned}
$$

Equation (B.13) is not exactly in the form most often found in the literature. Usually the orbit distortion $\Delta x$ is given for the particle whose trajectory is characterized by $x=0$ and $y=0$, solutions of the homogeneous equation. However, if the particle initially has $x \neq 0, \dot{x}^{\prime} \neq 0$, then the above-specified solution cannot characterize the trajectory of such initial conditions. For such particles, the second term on the right-hand side contributes to the orbit distortion over and above the contributions to the central trajectory. For completion these terms are included in Eq. (B.13)

In the next step we reparameterize Eq. (B.13) and Eq. (B.14) by assuming that $\Delta x=W U(\phi)$ and $\Delta D=W V(\phi)$. Since both equations are formally of a similar structure, we concentrate on Eq. (B.13):

$$
\begin{aligned}
& \Delta x^{\prime}=U W^{\prime}+\left(\frac{1}{\nu W}\right) \frac{d U}{d \phi} \\
& \Delta x^{\prime \prime}=U W^{\prime \prime}-\left(\frac{1}{\nu^{2} W^{3}}\right) \frac{d^{2} U}{d \phi^{2}}
\end{aligned}
$$

Recall that $W$ satisfies

$$
W^{\prime \prime}+K W=W^{-3}
$$

Using these relations and Eq. (B.15) for Eq. (B.13) and the analogous relations in Eq. (B.14) we get

$$
\begin{aligned}
& \frac{d^{2} U}{d \phi^{2}}+\nu^{2} U=\nu^{2} \beta^{3 / 2}\left[f_{\text {distort }}+\left\{\delta k_{\beta}-\frac{\Delta p}{p}\left(2 \rho^{-2}-k\right)\right\} x\right], \\
& \frac{d^{2} V}{d \phi^{2}}+\nu^{2} V=\nu^{2} \beta^{3 / 2}\left[f_{\text {dispers }}+\left\{\delta k_{\beta}-\frac{\Delta p}{p}\left(2 \rho^{-2}-k\right)\right\} D\right] .
\end{aligned}
$$

Equation for changes in the $\beta$ function:

Notice that the quadrupole strength term in Eq. (B.13) and Eq. (B.14) is the same as that appearing in Eq. (B.9) and Eq. (B.10). Hence, the ascillation frequency and the oscillation amplitude of these equations is relevant to the solutions of the perturbed equations given above. The well-known solution of Eq. (B.9) has been stated in the sections on linear motion in equations (1.9) through (1.15). There we characterized the solutions in terms of the phase function $\psi$ and a function $W^{2}=\beta$ satisfying equations (1.12) through (1.15). Let us consider the homogeneous part of Eq. (B.4) and let the coefficient of the 
term linear in $\mathrm{x}$ be called $\tilde{K}$ and the corresponding amplitude function $\tilde{W}=W+\Delta W$. Then $\tilde{W}$, along with $\tilde{K}=K+\delta K$, satisfies an equation analogous to Eq. (1.12). Equation (1.12) satisfied by $\tilde{W}$ and $W$ is rewritten below.

$$
\tilde{W}^{\prime \prime}+\tilde{K} \tilde{W}=\tilde{W}^{-3}
$$

Inserting $\tilde{W}=W+\Delta W$ and $\tilde{K}=K+\delta K$ in Eq. (B.18) we have

$$
\begin{aligned}
\tilde{W}^{\prime} & =\left(1+\frac{\Delta W}{W}\right) W^{\prime}+W \frac{\Delta W^{\prime}}{W} \\
\tilde{W}^{\prime \prime} & =\left(1+\frac{\Delta W}{W}\right) W^{\prime \prime}+2 W^{\prime} \frac{\Delta W^{\prime}}{W}+W \frac{\Delta W^{\prime \prime}}{W}, \\
\tilde{W}^{-3} & =\left(1+\frac{\Delta W}{W}\right) W^{-3}\left(1+\frac{\Delta W}{W}\right)^{-4}, \\
\tilde{W}^{\prime \prime}+\tilde{K} \tilde{W} & =\left(1+\frac{\Delta W}{W}\right)\left\{W^{\prime \prime}+K W\right\}+2 W^{\prime} \frac{\Delta W^{\prime}}{W}+W \frac{\Delta W^{\prime \prime}}{W}+W\left(1+\frac{\Delta W}{W}\right) \delta K .
\end{aligned}
$$

The above relations and Eq. (B.15) imply that:

$$
W \frac{\Delta W^{\prime \prime}}{W}+2 W^{\prime} \frac{\Delta W^{\prime}}{W}+W\left(1+\frac{\Delta W}{W}\right) \delta K \simeq-4 W^{-3} \frac{\Delta W}{W}
$$

Changing the parameter $\mathrm{s}$ of the differentiation to the parameter given by the phase $\psi$, where $\psi^{\prime}=$ $W^{-2}=\nu \phi^{\prime}$, we get

$$
\begin{aligned}
\frac{\Delta W^{\prime}}{W} & =\frac{1}{\nu W^{2}} \frac{d}{d \phi} \frac{\Delta W}{W}, \\
W \frac{\Delta W^{\prime \prime}}{W} & =-\frac{2 W^{\prime}}{\nu W^{2}} \frac{d}{d \phi} \frac{\Delta W}{W}+\frac{1}{\nu^{2} W^{3}} \frac{d^{2}}{d \phi^{2}} \frac{\Delta W}{W} .
\end{aligned}
$$

These expressions imply that Eq. (B.19) can be written as

$$
\frac{d^{2}}{d \phi^{2}} \frac{\Delta W}{W}+4 \nu^{2} \frac{\Delta W}{W} \simeq-W^{4} \nu^{2} \delta K
$$

Recall that $W^{2}=\beta$ so that that $\frac{\Delta W}{W}=\frac{\Delta \beta}{2 \beta}$. If we use this relation in Eq. (B.20) we get the equation for $\frac{\Delta \beta}{\beta}$. We have thus obtained the equation governing the changes in the $\beta$ function. Notice that the fractional change of $\beta$ oscillates with twice the frequency of the unperturbed system. 


\section{Appendix C.}

\section{Solutions to the Linearized Equations of Motion}

In Appendix B we found that the linearized equations satisfied by the orbit distortions $\frac{\Delta x}{\sqrt{\beta}}$, orbit dispersions $\frac{\Delta D}{\sqrt{\beta}}$ and $\frac{\delta \beta}{\beta}$, satisfy the inhomogeneous harmonic oscillator equations in appropriate coordinate systems. Hence the form of their solution is similar. For the sake of completion, we obtain the solution to the generalized form of the equation even though the solutions to these equations are well known. Let us consider an equation of the following form:

$$
\frac{d^{2} Z}{d \phi^{2}}+\hat{\nu}^{2} Z=F
$$

where $Z$ stands for $U$ or $V$ or $\frac{\Delta W}{W}$ and $\mathrm{F}$ could be any of the terms appearing on the right-hand side of the corresponding equations obtained in Appendix B. Since we are considering circular accelerators, the source terms on the right-hand side are periodic with periodicity equal to a revolution around the ring. We are interested in the closed orbit solutions, i.e we want $Z(\phi)=Z(\phi+2 \pi)$. We use the Green function method to solve Eq. (C.1). Let $G\left(\phi-\phi_{1}\right)$ be the Green function for the above equation, satisfying:

$$
\frac{d^{2} G\left(\phi-\phi_{1}\right)}{d \phi^{2}}+\hat{\nu}^{2} G\left(\phi-\phi_{1}\right)=\delta\left\{\hat{\nu}\left(\phi-\phi_{1}\right)\right\}
$$

Notice that for $\phi>\phi_{1}$ and $\phi<\phi_{1}, G\left(\phi-\phi_{1}\right)$ satisfies a linear homogeneous equation with constant coefficient. The two general linearly independent solutions to such an equation are $e^{i \hat{v}\left(\phi-\phi_{1}\right)}$ and $e^{-i \hat{\nu}\left(\phi-\phi_{1}\right)}$ The particular solution to the equation is some linear combination of these solutions. Let $C_{<}$and $G_{>}$be the form of the linear combination of the two general solutions in the regions denoted by $0<\phi<\phi_{1}$ and $\phi_{1}<\phi<2 \pi$, respectively. We want the solution to be such that at $\left(\phi=\phi_{1}\right) G_{<}=G_{>}$ and $\frac{d G_{<}}{d \phi}-\frac{d G_{>}}{d \phi}=1$. In addition to the conditions of continuity of the solution and discontinuity of its derivative at the source of the error, we want the solution and its derivative to be continuous at $(\phi=0)$ in a total of four conditions. Therefore, in each region of $\phi$ where the homogeneous equation is satisfied we can write $G_{<}$and $G_{>}$in the following form:

$$
\begin{aligned}
& G_{<}=c_{1} e^{i \hat{\nu}\left(\phi-\phi_{1}\right)}+c_{2} e^{-i \hat{\nu}\left(\phi-\phi_{1}\right)}, \\
& G_{>}=d_{1} e^{i \hat{\nu}\left(\phi-\phi_{1}\right)}+d_{2} e^{-\imath \hat{\nu}\left(\phi-\phi_{1}\right)} .
\end{aligned}
$$

Using the conditions of continuity of the Green function at $\phi_{1}$ and discontinuity of the derivative of the Green function at $\phi_{1}$, we find that

$$
\left(c_{1}-d_{1}\right)=-\left(c_{2}-d_{2}\right) ; \quad\left(c_{2}-d_{2}\right)-\left(c_{1}-d_{1}\right)=\left(\frac{1}{\imath \hat{\nu}}\right) .
$$

The above two conditions imply that

$$
\left(c_{1}-d_{1}\right)=\left(\frac{-1}{2 \imath \hat{\nu}}\right) ; \quad\left(c_{2}-d_{2}\right)=\left(\frac{1}{2 \imath \hat{\nu}}\right) .
$$


If we call $G_{s}=-\frac{1}{\hat{\nu}} \sin \left[\hat{\nu}\left(\phi-\phi_{1}\right)\right]$ in the region, $0 \leq<\leq \phi_{1}$, and $G_{s}=0$ in the region, $\phi_{1}<\phi \leq 2 \pi$, and use Eq. (C.5) and Eq. (C.6), we can write

$$
G\left(\phi-\phi_{1}\right)=G_{s}+d_{1} e^{2 \hat{\nu}\left(\phi-\phi_{1}\right)}+d_{2} e^{-2 \hat{\nu}\left(\phi-\phi_{1}\right)}
$$

The continuity of the Green function and its derivative at $\phi=0 \bmod (2 \pi)$ imply $d_{1}=\frac{z}{2 \bar{\nu}\left(e^{2 /\left(\phi-\phi_{1}\right)}-1\right)}$ and $d_{2}=\frac{-2}{2 \nu\left(e^{\left.-2 \tilde{L} \phi-\phi_{1}\right)-1}\right)}$. These in turn imply that:

$$
G\left(\phi-\phi_{1}\right)=\frac{\cos \left\{\hat{\nu}\left(\phi-\phi_{1}\right)\right\}}{2 \hat{\nu} \sin \pi \hat{\nu}}
$$

Given the Green function for Eq. (C.1) we can construct the solution to the original equation in the usual way. In the following, we write down the solution to three equations derived in Appendix B:

$$
\begin{aligned}
\frac{\delta x}{\sqrt{\beta}} & =\frac{1}{2 \nu \sin \pi \nu} \int_{\phi}^{\phi+2 \pi} F\left(\phi_{1}\right) \cos \left\{\nu\left(\phi-\phi_{1}+\pi\right)\right\} d \phi_{1}, \\
\frac{\Delta D}{\sqrt{\beta}} & =\frac{1}{2 \nu \sin \pi \nu} \int_{\phi}^{\phi+2 \pi} F\left(\phi_{1}\right) \cos \left\{\nu\left(\phi-\phi_{1}+\pi\right)\right\} d \phi_{1}, \\
\frac{\delta \beta}{\beta} & =\frac{1}{4 \nu \sin 2 \pi \nu} \int_{\phi}^{\phi+2 \pi} F\left(\phi_{1}\right) \cos \left\{2 \nu\left(\phi-\phi_{1}+\pi\right)\right\} d \phi_{1} .
\end{aligned}
$$

Recall that true errors are the driving terms in the harmonic oscillator with constant frequency. For such an oscillator there exists an invariant of motion which characterizes the amplitude of the motion. This invariant, say $I$, is given by

$$
I=Z^{2}+\frac{1}{\nu^{2}}\left(\frac{d Z}{d \phi}\right)^{2}
$$

I is similar to the total energy of a simple harmonic oscillator. Using Eq. (C.9), Eq. (C.10), and Eq. (C.11) and their derivatives in Eq. (C.12) successively we obtain the invariants we have stated earlier:

$$
\begin{aligned}
& I_{\delta x}=\left(\frac{1}{2 \nu \sin (\pi \nu)}\right)^{2} \int_{\phi}^{\phi+2 \pi} d \phi_{2} \int_{\phi}^{\phi+2 \pi} d \phi_{1} F\left(\phi_{1}\right) F\left(\phi_{2}\right) \cos \left[\nu\left(\phi_{2}-\phi_{1}\right)\right], \\
& I_{\delta D}=\left(\frac{1}{2 \nu \sin (\pi \nu)}\right)^{2} \int_{\phi}^{\phi+2 \pi} d \phi_{2} \int_{\phi}^{\phi+2 \pi} d \phi_{1} F\left(\phi_{1}\right) F\left(\phi_{2}\right) \cos \left[\nu\left(\phi_{2}-\phi_{1}\right)\right], \\
& I_{\delta \beta}=\left(\frac{1}{4 \nu \sin (2 \pi \nu)}\right)^{2} \int_{\phi}^{\phi+2 \pi} d \phi_{2} \int_{\phi}^{\phi+2 \pi} d \phi_{1} F\left(\phi_{1}\right) F\left(\phi_{2}\right) \cos \left[2 \nu\left(\phi_{2}-\phi_{1}\right)\right]
\end{aligned}
$$

where $F(\phi)$ denotes the right-hand side of the equations written in Appendix B. Also note that we can write $Z \simeq \frac{I}{\sqrt{2}} \sin \hat{\nu} \phi$ and $\frac{d Z}{d \phi} \simeq \frac{I}{\sqrt{2}} \cos \hat{\nu} \phi$.

\section{Approximations to a discreet error distribution:}

In order to simplify the above expressions for applications to circular accelerators we use the fact that the magnetic elements, hence the error sources, are discreetly distributed around the ring. Hence the above integrals contribute only at the position of the magnetic elements considered. Let us further assume that the errors and the beta functions are constant over the length of the magnetic element under consideration. We further assume that the term $\cos \hat{\nu}\left(\phi_{i}-\phi_{j}\right)$ does not change significantly over 
the length of the magnetic element. With these assumptions and the fact that $d \phi=\left(\frac{1}{\nu \beta}\right) d s=\left(\frac{1}{\nu \beta}\right) d L$, where $L$ is the length of the magnetic element, we obtain:

$$
\begin{aligned}
& I_{\delta x}=\left(\frac{1}{2 \nu \sin (\pi \nu)}\right)^{2} \sum_{i=\left(\text { element } t_{j}=\left(\text { element } t_{j}\right.\right.}^{i=\left(\text { element }_{n} j=\left(\text { element }_{\lambda}\right.\right.}\left[\frac{L_{i} F\left(\phi_{i}\right)}{\nu \beta_{i}} \frac{L_{j} F\left(\phi_{j}\right)}{\nu \beta_{j}} \cos \left[\nu\left(\phi_{i}-\phi_{j}\right)\right]\right],
\end{aligned}
$$

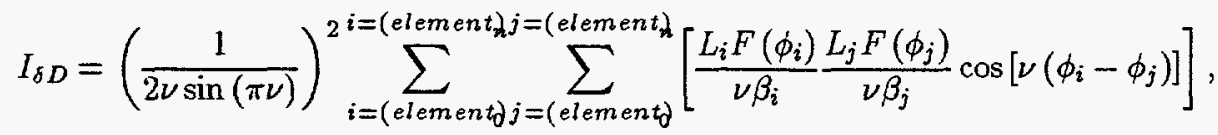

$$
\begin{aligned}
& I_{\delta \beta}=\left(\frac{1}{4 \nu \sin (2 \pi \nu)}\right)^{2} \sum_{i=\left(\text { element } t_{j}\right.}^{2=\left(\text { element } t_{j}\right.} \sum_{(\text {elent }}^{j=\left(\text { element } t_{n}\right.}\left[\frac{L_{i} F\left(\phi_{i}\right)}{\nu \beta_{i}} \frac{L_{j} F\left(\phi_{j}\right)}{\nu \beta_{j}} \cos \left[2 \nu\left(\phi_{i}-\phi_{j}\right)\right]\right] \text {, }
\end{aligned}
$$

where $\phi_{i}$ is the phase at the i'th element. Let $F_{j}$ denote $F\left(\phi_{j}\right)$. To apply the above to Eq. (B.16), Eq. (B.17), and Eq. (B.20) stated in Appendix B, we define $F_{i}=\nu^{2} \beta^{3 / 2} f_{i}$ for orbit distortion and $F_{i}=\nu^{2} \beta^{2} \delta K_{i}$ for $\delta \beta$. Using these substitutions we can write the above expressions in the following forms:

$$
\begin{aligned}
& I_{\delta x}=\left(\frac{1}{2 \sin (\pi \nu)}\right)^{2} \sum_{i=(\text { element } j}^{i=\left(\text { element } t_{h} j=(\text { elemement }\right.} \sum_{\text {elem }_{j}}\left[L_{i} \sqrt{\beta_{i}} f_{i} L_{j} \sqrt{\beta_{j}} f_{j} \cos \left[\nu\left(\phi_{i}-\phi_{j}\right)\right]\right], \\
& I_{\delta D}=\left(\frac{1}{2 \sin (\pi \nu)}\right)^{2} \sum_{i=(\text { element } j}^{i=\left(\text { element } t_{n} j=(\text { element }\right.} \sum_{j}^{\text {elent }}\left[L_{i} \sqrt{\beta_{i}} f_{i} L_{j} \sqrt{\beta_{j}} f_{j} \cos \left[\nu\left(\phi_{i}-\phi_{j}\right)\right]\right] \text {, }
\end{aligned}
$$

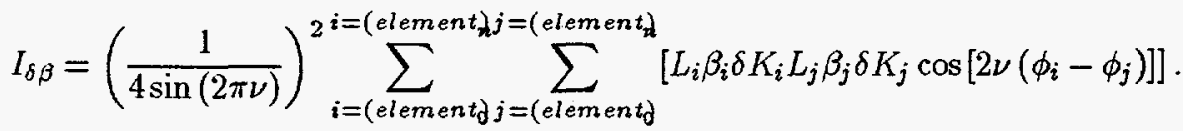

The tabular form of $f_{i}$ in the above expressions for the invariants for orbit distortion, dispersion, and the $\delta \beta$ are given below.

Table C.1: Source and Form of $f$ for Orbit Distortion:

\begin{tabular}{|c|c|c|c|c|}
\hline Magnet type & $f$ & $f$ & $f$ & $f$ \\
\hline & & & & \\
\hline Dipole & $\frac{\Delta B}{B \rho}$ & $\frac{\Delta B^{\prime}}{B \rho} x$ & $\frac{2 \Delta B}{B \rho^{2}} x$ & $\frac{2 \Delta p}{\rho^{2} p}$ \\
Quadrupole & $k \Delta x$ & $k \frac{\Delta B^{\prime}}{B^{\prime}} x$ & $\frac{2 k \Delta x}{\rho} x$ & $k x \frac{\Delta P}{p}$ \\
Sextupole & & & $s x \Delta x$ & \\
\hline
\end{tabular}


Table C.2: Source and Form of $f$ for Orbit Dispersion:

\begin{tabular}{|c|c|c|c|c|c|c|}
\hline Magnet type & $f$ & $f$ & $f$ & $f$ & $f$ & $f$ \\
\hline & & & & & & \\
\hline Dipole & $\frac{\Delta B}{B \rho}$ & $\frac{\Delta B^{\prime}}{B \rho} D$ & $\frac{2 \Delta B}{B^{2}} D$ & & & $\frac{2 \Delta p}{\rho^{2} p} D$ \\
Quadrupole & $k \Delta x$ & $k \sqrt{\beta} \frac{\Delta x}{\sqrt{\beta}}$ & $k \frac{\Delta B^{\prime}}{B^{\prime}} D$ & $\frac{2 k \Delta x}{\rho} D$ & $\frac{2 k \sqrt{\beta}}{\rho} \frac{\Delta x}{\sqrt{\beta}}$ & $k D \frac{\Delta P}{p}$ \\
Sextupole & & & $s D \sqrt{\beta} \frac{\delta x}{\sqrt{\beta}}$ & $s D \Delta x$ & & \\
\hline
\end{tabular}

Table C.3: Source and Form of $\delta K$ for $\frac{\delta . \beta}{\beta}$ and $\delta \nu$ :

\begin{tabular}{|c|c|c|c|}
\hline Magnet type & $\delta K$ & $\delta K$ & $\delta K$ \\
\hline Dipole & $\frac{\Delta B^{\prime}}{B \rho}$ & $\frac{2 \Delta B}{B \rho^{2}}$ & \\
Quadrupole & $k \frac{\Delta B^{\prime}}{B^{\top}}$ & $\frac{2 k \sqrt{\beta}}{\rho} \frac{\delta x}{\sqrt{\beta}}$ & $\frac{2 k \Delta x}{\rho}$ \\
Sextupole & $s \sqrt{\beta} \frac{\delta x}{\sqrt{\beta}}$ & $s \Delta x$ & \\
\hline
\end{tabular}

Table C.4: Source and Form of $\delta \xi=\frac{\delta \nu}{\Delta p / p}$;

\begin{tabular}{|c|c|c|c|c|c|c|c|c|}
\hline Magnet type & $\delta K$ & $\delta K$ & $\delta K$ & $\delta K$ & $\delta K$ & $\delta K$ & $\delta K$ & \\
\hline & & & & & & & & \\
\hline Dipole & $\frac{\Delta B^{\prime}}{B^{\rho}}$ & $\frac{2 \Delta B}{B \rho^{2}}$ & $D \frac{\Delta B^{\prime \prime}}{B \rho}$ & $\frac{2}{\rho^{2}}$ & & & & \\
Quadrupole & $k \frac{\Delta B^{\prime}}{B^{\prime}}$ & $k D \frac{\Delta B^{\prime \prime}}{B^{\prime}}$ & $\frac{2 k \sqrt{\beta}}{\rho} \frac{\delta x}{\sqrt{\beta}}$ & $\frac{2 k \Delta x}{\rho}$ & $\frac{2 k}{\rho} D$ & $\frac{2 k \sqrt{\beta}}{\rho} \frac{\delta D}{\sqrt{\beta}}$ & $2 k D \frac{\Delta B^{\prime}}{B^{\prime}}$ & $k$ \\
Sextupole & $s \sqrt{\beta} \frac{\delta x}{\sqrt{\beta}}$ & $s \Delta x$ & $s D \delta \beta$ & $s \sqrt{\beta} \frac{\delta D}{\sqrt{\beta}}$ & $s \frac{\Delta B^{\prime \prime}}{B^{\prime \prime}}$ & & & \\
\hline
\end{tabular}




\section{Appendix D.}

\section{Application to the APS Injector Synchrotron}

In this appendix we apply the results from the linear theory as given in the previous parts to specify the tolerance budget for the injector synchrotron ${ }^{6}$. The results and conclusions of the calculations as given by the linear theory are given in Tables D.1 through D.22, in terms of the error sources. If we know the accuracy of the beam position monitors and the accuracy within which we can place the magnetic elements then these tables provide us with reasonable upper limits on the error tolerances.

We expect that the survey alignment accuracy is approximately $2 \times 10^{-4}$ meters, both in the horizontal and the vertical directions. Also the BPM accuracy is expected to be $10^{-4}$ meters. In order to establish tolerance limits of errors we assume that these accuracies.are met.

Consider the orbit distortion tables, Table D.1 and Table D.11 for horizontal and vertical motion, respectively. With rms values for $\Delta x=2 \times 10^{-4}$ meters, we find $\left\langle\frac{\delta x}{\sqrt{\beta_{x}}}\right\rangle=1.4 \times 10^{-3} \sqrt{\text { meters }}$ and $<\frac{\delta y}{\sqrt{\beta}_{y}}>=1.5 \times 10^{-3} \sqrt{\text { meters }}$. With mean $\beta_{x} \sim 8.3$ meters and $\beta_{y} \sim 8.8$ meters, $<\delta x>\sim 4.1 \mathrm{~mm}$ and $<\delta y>\sim 4.5 \mathrm{~mm}$. Without any orbit correction, we demand that other sources of the orbit distortion may contribute at most the same amount. This amount of orbit distortion due to the dipole field error in dipole magnets implies that $\frac{\Delta B}{B} \sim 10^{-} 3$ and $\Delta \theta \sim 10^{-}-3$. Hence orbit correction is necessary. We expect to keep the orbit distortion to about $.1 \mathrm{~mm}$ by using orbit correctors. After correction $\left\langle\frac{\delta x}{\sqrt{\beta_{x}}}>\sim .03 \mathrm{~mm}\right.$ and $\left\langle\frac{\delta y}{\sqrt{\beta_{y}}}>\sim .034 \mathrm{~mm}\right.$. It is clear from Tables D.1 and D.6 that the contributions from other sources is a factor of three or less than the orbit achieved after orbit correction. Therefore, these two tables do not give rise to any further restrictions on other multipoles. Henceforth we will assume that orbit correction has been implemented and $\left\langle\frac{\delta x}{\sqrt{\beta_{x}}}>\sim .03 \times 10^{-} 3 \sqrt{\text { meters }}\right.$ and $\left\langle\frac{\delta y}{\sqrt{\beta_{y}}}>\sim .034 \times 10^{-} 3 \sqrt{\text { meters }}\right.$.

We consider next Table D.3. In view of the assumptions about $\Delta x$ and $\left\langle\frac{\delta x}{\sqrt{\beta_{x}}}>\right.$ from survey alignment and orbit correction criteria, respectively, we find from Table D.3 that the contributions to $<\frac{\delta D}{\sqrt{\beta_{x}}}>$ from these sources in quadrupoles and sextupoles are $\left\langle\frac{\delta D}{\sqrt{\beta_{x}}}>_{q u a d} \sim 7.92 \times 10^{-4} \sqrt{\text { meters }}\right.$, $<\frac{\delta D}{\sqrt{\beta_{x}}}>_{\text {sex }} \sim 8.93 \times 10^{-4} \sqrt{\text { meters }},<\frac{\delta D}{\sqrt{\beta_{x}}}>_{q u a \sigma} \sim 1.4 \times 10^{-3} \sqrt{\text { meters }}$, and $\left\langle\frac{\delta D}{\sqrt{\beta_{x}}}\right\rangle_{\text {sex }} \sim 1.7 \times$ $10^{-3} \sqrt{\text { meters }}$, respectively. We use the criterion that the contribution from other sources to $\left\langle\frac{\delta D}{\sqrt{\beta_{x}}}\right\rangle$ may be at most equal to $1.7 \times 10^{-3} \sqrt{\text { meters. }}$. This criterion gives the tolerance limits on some of the field components in dipoles and quadrupoles. The conclusions about tolerances drawn from Table D.3 are given in Table D.4.

Similar considerations shown in Table D.5, Table D.14, Table D.7, Table D.16, Table D.9, and Table D.18, while using values for $\Delta x,\left\langle\frac{\delta x}{\sqrt{\beta_{x}}}\right\rangle, \Delta y,\left\langle\frac{\delta y}{\sqrt{\beta_{y}}}\right\rangle$, and $\left\langle\frac{\delta D}{\sqrt{\beta_{x}}}\right\rangle$ obtained previously, give restrictions on the multipole moment errors in different magnetic elements. The conclusions obtained from each table are given separately in Table D.6, Table D.15, Table D.8, Table D.17, Table D.10, and Table D.19, respectively. The final conclusion about tolerances on different multipole components is obtained by comparing these different tables, and appears in Table D.20. In the end, Table D.21, 
and Table D.22 give the total change in each beam dynamical parameter, as obtained after using the estimated values.

Table D.1: Horizontal Orbit Distortion Calculations $\frac{\delta x}{\sqrt{\beta_{x}}}=\sqrt{\frac{I_{\delta x}}{2}}$.

\begin{tabular}{|c|c|c|c|}
\hline Error Source & Dipole & Quadrupole & Sextupole \\
\hline & $\sqrt{I_{\delta x}}$ & $\sqrt{I_{\delta x}}$ & $\sqrt{I_{\delta x}}$ \\
\hline$\frac{\Delta B}{B \rho}$ & $1.41 \frac{\Delta B}{B}$ & & \\
$\frac{\Delta B^{\prime}}{B \rho} x$ & $.0013 \frac{\Delta B^{\prime}}{B}$ & & \\
$\frac{2 \Delta B}{B \rho^{2}} x$ & $.0008 \frac{\Delta B}{B}$ & & \\
$\frac{2 \Delta p}{\rho^{2} p} x$ & $.000053 \frac{\Delta p}{p}$ & & \\
\hline$k \Delta x$ & & $6.88 \Delta x$ & \\
$k \frac{\Delta B^{\prime}}{B^{\prime}} x$ & & $.0095 \frac{\Delta B^{\prime}}{B^{\prime}}$ & \\
$\frac{2 k \Delta x}{\rho} x$ & & $.0057 \Delta x$ & \\
$k x \frac{\Delta P}{p}$ & & $.0036 \frac{\Delta P}{p}$ & \\
\hline$s x \Delta x$ & & & $.013 \Delta x$ \\
\hline
\end{tabular}

From a survey accuracy of $\Delta x \sim 2 \times 10^{-4} m$ we find that $\left\langle\frac{\delta x}{\sqrt{\beta}}>\sim 1.6 \times 10^{-3} \sqrt{m}\right.$.

Table D.2: Tolerance Limits Due to Orbit Distortion in the Horizontal Direction. Assumed $<\frac{\delta x}{\sqrt{\beta}_{x}}>\sim 1.6 \times 10^{-3} \sqrt{m}$ and $\Delta x \sim 2 \times 10^{-4} m$

\begin{tabular}{|c|c|c|}
\hline & $\left\langle\frac{\Delta B}{B}\right\rangle_{\text {dipole }}$ & $\frac{\Delta B^{\prime}}{B}$ \\
\hline & & \\
\hline Tolerance & $10^{-3}$ & 1.1 per meter \\
\hline
\end{tabular}


Table D.3: Horizontal Dispersion Calculations $\frac{\delta D}{\sqrt{\beta_{x}}}=\sqrt{\frac{I_{\delta D}}{2}}$.

\begin{tabular}{|c|c|c|c|}
\hline Error Source & Dipole & Quadrupole & Sextupole \\
\hline & $\sqrt{T_{\delta D}}$ & $\sqrt{T_{\delta D}}$ & $\sqrt{I_{\delta D}}$ \\
\hline$\frac{\Delta B}{B \rho}$ & $1.41 \frac{\Delta B}{B}$ & & \\
$\frac{\Delta B^{\prime}}{B \rho} D$ & $.8 \frac{\Delta B^{\prime}}{B}$ & & \\
$\frac{2 \Delta B}{B \rho^{2}} D$ & $.05 \frac{\Delta B}{B}$ & & \\
$\frac{2 \Delta p}{\rho^{2} p} D$ & $.06 \frac{\Delta p}{p}$ & & \\
\hline$k \Delta x$ & & $6.88 \Delta x$ & \\
$k \sqrt{\beta} \frac{\Delta x}{\sqrt{\beta}}$ & & $26.4 \frac{\Delta x}{\sqrt{\beta}}$ &. \\
$\frac{2 k \Delta x}{\rho} D$ & & $.33 \Delta x$ & \\
$\frac{2 k \sqrt{\beta}}{\rho} \frac{\Delta x}{\sqrt{\beta}}$ & & $1.31 \frac{\Delta x}{\sqrt{\beta}}$ & \\
$k \frac{\Delta B^{\prime}}{B^{\prime}} D$ & & $5.49 \frac{\Delta B^{\prime}}{B^{\prime}}$ & \\
$k D \frac{\Delta P}{p}$ & & $2.46 \frac{\Delta P}{p}$ & \\
\hline$s D \Delta x$ & & & $8.31 \Delta x$ \\
$s D \sqrt{\beta} \frac{\Delta x}{\sqrt{\beta}}$ & & & $29.75 \frac{\Delta x}{\sqrt{\beta}}$ \\
\hline
\end{tabular}

Table D.4: vTolerance Limits from Dispersion Calculations in the Horizontal Direction. Assumed corrected orbit with $<\frac{\delta x}{\sqrt{\hat{\beta}_{x}}}>\sim 3 \times 10^{-5} \sqrt{m}$ and $\Delta x=2 \times 10^{-4} \mathrm{~m}$; this implies $\frac{\delta D}{\sqrt{\beta_{x}}} \sim 1.7 \times 10^{-3} \sqrt{m}$.

\begin{tabular}{|l|c|c|c|}
\hline & $\left.<\frac{\Delta B}{B}\right\rangle_{D}$ & $\left.<\frac{\Delta B^{\prime}}{B}\right\rangle_{D}$ & $\left.<\frac{\Delta B^{\prime}}{B^{\prime}}\right\rangle_{Q}$ \\
\hline & & & \\
\hline Tolerance & $1.2 \times 10^{-3}$ & $2.1 \times 10^{-3} M^{-1}$ & $3.1 \times 10^{-4}$ \\
\hline
\end{tabular}


Table D.5: $\frac{\delta \beta_{x}}{\beta_{x}}$ Calculations $\frac{\delta \beta_{x}}{\sqrt{\beta_{x}}}=\sqrt{2 I_{\delta \beta}}$

\begin{tabular}{|c|c|c|c|}
\hline Error Source & Dipole & Quadrupole & Sextupole \\
\hline & $\sqrt{I_{\delta \beta_{x}}}$ & $\sqrt{I_{\delta \beta_{x}}}$ & $\sqrt{I_{\delta \beta_{x}}}$ \\
\hline$\frac{\Delta B^{\prime}}{\beta^{\rho}}$ & $2.45 \frac{\Delta B^{\prime}}{B}$ & & \\
$\frac{2 \Delta B}{B \rho^{2}}$ & $.15 \frac{\Delta B}{B}$ & & \\
\hline$k \frac{\Delta B^{\prime}}{B^{\prime}}$ & & $18.15 \frac{\Delta B^{\prime}}{B^{\prime}}$ & \\
$\frac{2 k \Delta x}{\rho}$ & & $1.09 \Delta x$ & \\
$\frac{2 k \sqrt{\beta}}{\rho} \frac{\Delta x}{\sqrt{\beta}}$ & & $4.33 \frac{\delta x}{\sqrt{\beta_{x}}}$ & \\
\hline$s \Delta x$ & & & $24.77 \Delta x$ \\
$s \sqrt{\beta} \frac{\Delta x}{\sqrt{\beta}}$ & & & $90.2 \frac{\Delta x}{\sqrt{\beta}}$ \\
\hline
\end{tabular}

Table D.6: Tolerance Limits from $\frac{\delta \beta_{x}}{\beta_{x}}$, using $\Delta x \sim 2 \times 10^{-4} \mathrm{~m}$ and $\frac{\delta x}{\sqrt{\beta_{x}}} \sim 3 \times 10^{-5} \sqrt{m}$. We have used $\frac{\delta \beta_{x}}{\beta_{x}} \sim 2.7 \times 10^{-3}$.

\begin{tabular}{|l|c|c|}
\hline & $<\frac{\Delta B^{\prime}}{B}>_{D}$ & $<\frac{\Delta_{B^{\prime}}}{B^{\prime}}>_{Q}$ \\
\hline & & \\
\hline Tolerance & $1.1 \times 10^{-3} M^{-1}$ & $1.4 \times 10^{-4}$ \\
\hline
\end{tabular}


Table D.7: $\delta \nu_{x}$ Calculations.

\begin{tabular}{|c|c|c|c|}
\hline Error Source & Dipole & Quadrupole & Sextupole \\
\hline & $\delta \nu_{x}$ & $\delta \nu_{x}$ & $\delta \nu_{x}$ \\
\hline$\frac{\Delta B^{\prime}}{B \rho}$ & $.78 \frac{\Delta B^{\prime}}{B}$ & & \\
$\frac{2 \Delta B}{B \rho^{2}}$ & $.05 \frac{\Delta B}{B}$ & & \\
\hline$k \frac{\Delta B^{\prime}}{B^{\prime}}$ & & $5.77 \frac{\Delta B^{\prime}}{B^{\prime}}$ & \\
$\frac{2 k \Delta x}{\rho}$ & & $.35 \Delta x$ & \\
$\frac{2 k \sqrt{\beta}}{\rho} \frac{\Delta x}{\sqrt{\beta}}$ & & $1.38 \frac{\delta x}{\sqrt{\beta x}}$ & \\
\hline$s \Delta x$ & & & $7.87 \Delta x$ \\
$s \sqrt{\beta} \frac{\Delta x}{\sqrt{\beta}}$ & & & $28.66 \frac{\Delta x}{\sqrt{\beta}}$ \\
\hline
\end{tabular}

Table D.8: Tolerance Limit using $\delta \nu_{x}$, $\Delta x \sim 2 \times 10^{-4}$, and $\frac{\delta x}{\sqrt{\beta_{x}}} \sim 3 \times 10^{-5} \sqrt{m}$.

\begin{tabular}{|c|c|c|}
\hline & $\left\langle\frac{\Delta B^{\prime}}{B}\right\rangle_{D}$ & $<\frac{\Delta B^{\prime}}{B^{\prime}}>_{Q}$ \\
\hline & & \\
\hline Tolerance & $2.1 \times 10^{-3} M^{-1}$ & $2.8 \times 10^{-4}$ \\
\hline
\end{tabular}


Table D.9: $\delta \xi_{x}=\frac{\delta \nu}{\Delta p / p}$ Calculations.

\begin{tabular}{|c|c|c|c|}
\hline Error Source & Dipole & Quadrupole & Sextupole \\
\hline & $\delta \xi_{x}$ & $\delta \xi_{x}$ & $\delta \xi_{x}$ \\
\hline$\frac{\Delta B^{\prime}}{B \rho}$ & $.78 \frac{\Delta B^{\prime}}{B}$ & & \\
\hline$\frac{2 \Delta B}{B \rho^{2}}$ & $.05 \frac{\Delta B}{B}$ & & \\
\hline$D \frac{\Delta B^{\prime \prime}}{B \rho}$ & $.3 \frac{\Delta B^{\prime \prime}}{B}$ & & \\
\hline$\frac{2}{\rho^{2}}$ & & & \\
\hline$k \frac{\Delta B^{\prime}}{B^{\prime}}$ & & $5.77 \frac{\Delta B^{\prime}}{B^{\prime}}$ & \\
\hline $2 k D \frac{\Delta B^{\prime}}{B^{\prime} \rho}$ & & $.14 \frac{\Delta B^{\prime}}{B^{\prime}}$ & - \\
\hline$k D \frac{\Delta B^{\prime \prime}}{B^{\prime}}$ & & $2.38 \frac{\Delta B^{\prime \prime}}{B^{\top}}$ & \\
\hline$\frac{2 k \Delta x}{\rho}$ & & $.35 \Delta x$ & \\
\hline$\frac{2 k \sqrt{\beta}}{\rho} \frac{\Delta x}{\sqrt{\beta}}$ & & $1.38 \frac{\Delta x}{\sqrt{\beta}}$ & . \\
\hline$\frac{2 k \sqrt{\beta}}{\rho} \frac{\Delta D}{\sqrt{\beta}}$ & & $1.38 \frac{\Delta D}{\sqrt{\beta}}$ & \\
\hline$k$ & & .07 & \\
\hline$s \frac{\Delta B^{\prime \prime}}{B^{\prime \prime}}$ & & & $4.59 \frac{\Delta B^{\prime \prime}}{B^{\prime \prime}}$ \\
\hline$s \Delta x$ & & & $7.87 \Delta x$ \\
\hline$s \sqrt{\beta} \frac{\Delta x}{\sqrt{\beta}}$ & & & $28.66 \frac{\Delta x}{\sqrt{\beta}}$ \\
\hline$s \sqrt{\beta} \frac{\Delta D}{\sqrt{\beta}}$ & & & $90.2 \frac{\Delta D}{\sqrt{\beta}}$ \\
\hline
\end{tabular}

Table D.10: Tolerance Limits from $\delta \xi_{x}$,

using $\Delta x \sim 2 \times 10^{-4} \mathrm{~m}, \frac{\delta x}{\sqrt{\beta_{x}}} \sim 3 \times 10^{-5} \sqrt{m}$,

and $<\frac{\delta D}{\sqrt{\beta_{x}}}>\sim 2.5 \times 10^{-3} \sqrt{m}$;

implying that $\delta \xi_{x} \leq 2.3 \times 10^{-1}$.

\begin{tabular}{|l|c|c|c|c|c|}
\hline & $<\frac{\Delta B^{\prime}}{B}>_{D}$ & $<\frac{\Delta B^{\prime}}{B}>_{D}$ & $<\frac{\Delta B^{\prime}}{B^{\prime}}>_{Q}$ & $<\frac{\Delta B^{\prime}}{B^{\prime}}>_{Q}$ & $<\frac{\Delta B^{\prime}}{B^{\prime \prime}}>_{S}$ \\
\hline & & & & & \\
\hline Tolerance & $4.1 \times 10^{-1} m^{-1}$ & $1.1 m^{-2}$ & $5.5 \times 10^{-2}$ & $1.3 \times 10^{-1} m^{-1}$ & $6.97 \times 10^{-2}$ \\
\hline
\end{tabular}


Table D.11: Vertical Orbit Distortion Calculations $\frac{\delta y}{\sqrt{\beta_{y}}}=\sqrt{\frac{I_{\delta y}}{2}}$.

\begin{tabular}{|c|c|c|c|}
\hline Error Source & Dipole & Quadrupole & Sextupole \\
\hline & $\sqrt{I_{\delta y}}$ & $\sqrt{I_{\delta y}}$ & $\sqrt{I_{\delta y}}$ \\
\hline$\Delta \theta$ & $1.74 \Delta \theta$ & & \\
\hline$k \Delta y$ & & $7.6 \Delta y$ & \\
$k \frac{\Delta B^{\prime}}{B^{\prime}} x$ & & $.01 \frac{\Delta B^{\prime}}{B^{\prime}}$ & \\
$\frac{2 k \Delta y}{p} x$ & & $.0006 \Delta y$ & \\
$k x \frac{\Delta P}{p}$ & & $.011 \frac{\Delta P}{p}$ & \\
\hline$s x \Delta y$ & & & $.027 \Delta y$ \\
\hline
\end{tabular}

Table D.12: Tolerance Limits from Vertical Orbit Distortion. Used $<\frac{\delta y}{\sqrt{\beta_{y}}}>\sim 1.5 \times 10^{-3} \sqrt{m}$ and $\Delta y \sim 2 \times 10^{-4} \mathrm{~m}$.

\begin{tabular}{|l|c|}
\hline & $\left\langle\frac{\Delta B}{B}\right\rangle_{\text {dipole }} \Delta \theta$ \\
\hline & \\
\hline Tolerance & $10^{-3}$ \\
\hline
\end{tabular}

Table D.13: Dispersion Calculations in the Vertical Direction $\frac{\delta D}{\sqrt{\beta_{x}}}=\sqrt{\frac{I \delta x}{2}}$.

\begin{tabular}{|c|c|c|c|}
\hline Error Source & Dipole & Quadrupole & Sextupole \\
\hline & $\sqrt{I_{\delta D_{y}}}$ & $\sqrt{I_{\delta D_{y}}}$ & $\sqrt{I_{\delta D_{y}}}$ \\
\hline$k \Delta y$ & & $7.6 \Delta y$ & \\
\hline
\end{tabular}


Table D.14: $\frac{\delta \beta_{y}}{\beta_{y}}$ Calculation $\frac{\delta \beta_{y}}{\sqrt{\beta_{y}}}=\sqrt{2 I_{\delta \beta_{y}}}$.

\begin{tabular}{|c|c|c|c|}
\hline Error Source & Dipole & Quadrupole & Sextupole \\
\hline & $\sqrt{I_{\delta \beta_{y}}}$ & $\sqrt{I_{\delta \beta_{y}}}$ & $\sqrt{I_{\delta \beta_{y}}}$ \\
\hline$\frac{\Delta B^{\prime}}{B \rho}$ & $2.90 \frac{\Delta B^{\prime}}{B}$ & & \\
\hline$k \frac{\Delta B^{\prime}}{B^{\prime}}$ & & $17.5 \frac{\Delta B^{\prime}}{B^{\prime}}$ & \\
$\frac{2 k \Delta y}{\rho}$ & & $1.05 \Delta y$ & \\
$\frac{2 k \sqrt{\beta}}{\rho} \frac{\delta y}{\sqrt{\beta_{y}}}$ & & $4.17 \frac{\delta y}{\sqrt{\beta_{y}}}$ & \\
\hline$s \Delta y$ & & & $46.43 \Delta y$ \\
$s \sqrt{\beta} \frac{\delta y}{\sqrt{\beta_{y}}}$ & & & $167.93 \frac{\delta y}{\sqrt{\beta_{y}}}$ \\
\hline
\end{tabular}

Table D.15: Tolerance Limits from $\frac{\delta \beta_{y}}{\beta_{y}}$,

Assumed $\Delta y \sim 2 \times 10^{-4} m, \frac{\delta y}{\sqrt{\beta}_{y}} \sim 3.4 \times 10^{-5} \sqrt{m}$, and $\frac{\delta \beta_{y}}{\beta_{y}} \sim 9.3 \times 10^{-3}$.

\begin{tabular}{|l|c|c|}
\hline & $\left\langle\frac{\Delta B^{\prime}}{B}\right\rangle_{D}$ & $<\frac{\Delta B^{\prime}}{B^{\prime}}>_{Q}$ \\
\hline & & \\
\hline Tolerance & $3.2 \times 10^{-3} m^{-1}$ & $5.3 \times 10^{-4}$ \\
\hline
\end{tabular}

Table D.16: $\delta \nu_{y}$ Calculations.

\begin{tabular}{|c|c|c|c|}
\hline Error Source & Dipole & Quadrupole & Sextupole \\
\hline & $\delta \nu_{y}$ & $\delta \nu_{y}$ & $\delta \nu_{y}$ \\
\hline$\frac{\Delta B^{\prime}}{B \rho}$ & $.88 \frac{\Delta B^{\prime}}{B}$ & & \\
\hline$k \frac{\Delta B^{\prime}}{B^{\prime}}$ & & $5.29 \frac{\Delta B^{\prime}}{B^{\prime}}$ & \\
$\frac{2 k \Delta y}{\rho}$ & & $.32 \Delta y$ & \\
$\frac{2 k \sqrt{\beta}}{\rho} \frac{\delta y}{\sqrt{\beta_{y}}}$ & & $1.26 \frac{\delta y}{\sqrt{\beta_{y}}}$ & \\
\hline$s \Delta y$ & & & $14.05 \Delta y$ \\
$s \sqrt{\beta} \frac{\delta y}{\sqrt{\beta_{y}}}$ & & & $53.36 \frac{\delta y}{\sqrt{\beta_{y}}}$ \\
\hline
\end{tabular}


Table D.17: Tolerance Limit using $\delta v_{y}$, $\Delta y \sim 2 \times 10^{-4} m$, and $\frac{\delta y}{\sqrt{\beta_{y}}} \sim 3.4 \times 10^{-5} \sqrt{m}$.

\begin{tabular}{|l|c|c|}
\hline & $\left.<\frac{\Delta B^{\prime}}{B}\right\rangle_{D}$ & $<\frac{\Delta B^{\prime}}{B^{\prime}}>_{Q}$ \\
\hline & & \\
\hline Tolerance & $3.2 \times 10^{-3} m^{-1}$ & $5.3 \times 10^{-4}$ \\
\hline
\end{tabular}

Table D.18: $\delta \xi_{y}=\frac{\delta \nu}{\Delta p / p}$ Calculations.

\begin{tabular}{|c|c|c|c|}
\hline Error Source & Dipole & Quadrupole & Sextupole \\
\hline & $\delta \xi_{y}$ & $\delta \xi_{y}$ & $\delta \xi_{y}$ \\
\hline$\frac{\Delta B^{\prime}}{B^{\prime}}$ & $.88 \frac{\Delta B^{\prime}}{B}$ & & - \\
\hline$k \frac{\Delta B^{\prime}}{B^{\prime}}$ & & $5.29 \frac{\Delta B^{\prime}}{B^{\prime}}$ & \\
$\frac{2 k \Delta y}{\rho}$ & & $.32 \Delta y$ & \\
$\frac{2 k \sqrt{\beta}}{\rho} \frac{\delta y}{\sqrt{\beta_{y}}}$ & & $1.26 \frac{\delta y}{\sqrt{\beta_{y}}}$ & \\
\hline$s \frac{\Delta B^{\prime \prime}}{B^{\prime \prime}}$ & & & \\
$s \Delta y$ & & & $14.05 \Delta y$ \\
$s \sqrt{\beta} \frac{\delta y}{\sqrt{\beta_{y}}}$ & & & $53.36 \frac{\delta y}{\sqrt{\beta_{y}}}$ \\
\hline
\end{tabular}

Table D.19: Tolerance Limit using $\delta \xi_{y}$, $\Delta y \sim 2 \times 10^{-4} m$, and $\frac{\delta y}{\sqrt{\beta_{y}}} \sim 3.4 \times 10^{-5} \sqrt{m}$.

\begin{tabular}{|l|l|l|}
\hline & $<\frac{\Delta B^{\prime}}{B}>_{D}$ & $<\frac{\Delta B^{\prime}}{B^{\prime}}>_{Q}$ \\
\hline & & \\
\hline Tolerance & $3.2 \times 10^{-3} m^{-1}$ & $5.3 \times 10^{-4}$ \\
\hline
\end{tabular}

Table D.20: Summary of Tolerance Limit on Multipole Errors from Tables D.1 through D.19.

\begin{tabular}{|c|c|c|c|c|c|c|}
\hline & $<\frac{\Delta B}{B}>_{D}$ & $<\frac{\Delta B^{\prime}}{B}>_{D}$ & $<\frac{\Delta B^{\prime}}{B}>_{D}$ & $<\frac{\Delta B^{\prime}}{B^{\prime}}>_{Q}$ & $<\frac{\Delta B^{\prime}}{B^{\prime}}>_{Q}$ & $<\frac{\Delta B^{\prime}}{B^{\prime \prime}}>_{S}$ \\
\hline & & & & & & \\
\hline Tolerance & $10^{-3}$ & $2.1 \times 10^{-3} m^{-1}$ & $1.0 m^{-2}$ & $1.5 \times 10^{-3}$ & $.13 m^{-1}$ & .07 \\
\hline
\end{tabular}


Table D.21: Resulting Change in Beam Parameters, Horizontal Direction.

\begin{tabular}{|c|c|c|c|}
\hline$\left\langle\frac{\delta D}{\sqrt{\beta}_{x}}\right\rangle$ & $\left\langle\frac{\delta \beta_{x}}{\beta_{x}}\right\rangle$ & $\delta \nu_{x}$ & $\delta \xi_{x}$ \\
\hline $3.6 \times 10^{-3} \sqrt{m}$ & $6.4 \times 10^{-3}$ & $2.6 \times 10^{-3}$ & 0.51 \\
\hline
\end{tabular}

Table D.22: Resulting Change in Beam Parameters, Vertical Direction.

\begin{tabular}{|c|c|c|c|}
\hline$\left\langle\frac{\delta D}{\sqrt{\beta}_{y}}\right\rangle$ & $\left\langle\frac{\delta \beta_{y}}{\beta_{y}}\right\rangle$ & $\delta \nu_{y}$ & $\delta \xi_{y}$ \\
\hline & & & \\
\hline $1.5 \times 10^{-3} \sqrt{m}$ & $1.3 \times 10^{-2}$ & $3.9 \times 10^{-3}$ & $3.9 \times 10^{-3}$ \\
\hline
\end{tabular}

\section{References}

1. D. Edwards and M. Syphers, AIP Conference Proceedings No 184, Physics of Particle Accelerators, Fermi Lab Summer School, 1987.

2. E. D. Courant and H. S. Snyder, "Theory of Alternating Gradient Synchrotron," Ann.Phys. 3, 1 (1958).

3. Leo Michelotti, Introduction to Nonlinear Dynamics Arising from Magnetic Multipoles, 1984 U.S Summer School on High-energy Accelerators, Fermi National Laboratory, Batavia, Illinois, August $13-24,1984$.

4. A. J. Lichtenberg and M. A. Liberman, Regular and Stochastic Motion, Springer-Verlag, New York Heidelberg Berlin, (1983).

5. K. Steffen, Fundamentals of Accelerator Optics, Cern Accelerator School, Synchrotron Radiation And Free Electron Lasers, April 1989 , Editor S. Turner.

6. L. Teng, APS, private communication.

\section{DISCLAIMER}

This report was prepared as an account of work sponsored by an agency of the United States Ther Government nor any agency thereof, nor any of their Government. Neither the United States Government nor any agency the liability or responsiemployees, makes any warrant, exps, or usefulness of any information, apparatus, product, or bility for the accuracy, completeness, or usefulness of any information, apparatus, product, process disclosed, or represents that its use would not infringe privately owned rights. Reference herein to any specific commercial product, process, or service by trade name, trader recommanufacturer, or otherwise does not necessarily constitute or imply its endorsement, recommendation, or favoring by the United States Government or any agency thereof. The views and opinions of authors expressed herein do not necessarily state or reflect those of the United States Government or any agency thereof. 